\title{
Nonadiabatic Dynamics Algorithms with Only Potential Energies and Gradients: Curvature-Driven Coherent Switching with Decay of Mixing and Curvature-Driven Trajectory Surface Hopping
}

Yinan Shu, ${ }^{1, \S}$ Linyao Zhang, ${ }^{2,3, \S}$ Xiye Chen, ${ }^{2,3,}{ }^{*}$ Shaozeng Sun, ${ }^{2}$ Yudong Huang, ${ }^{3}$ and Donald G. Truhlar ${ }^{1, *}$

${ }^{1}$ Department of Chemistry and Supercomputing Institute, University of Minnesota, Minneapolis, MN 55455-0431, USA

${ }^{2}$ School of Energy Science and Engineering, Harbin Institute of Technology,

Harbin 150001, P. R. China

${ }^{3}$ School of Chemistry and Chemical Engineering, Harbin Institute of Technology, Harbin 150001, P. R. China

*Corresponding authors: truhlar@umn.edu, chenxiye@,hit.edu.cn

ABSTRACT. Direct dynamics by mixed quantum-classical nonadiabatic methods is an important tool for understanding processes involving multiple electronic states. Very often, the computational bottleneck of such direct simulation comes from electronic structure theory. For example, at every time step of a trajectory, nonadiabatic dynamics requires potential energy surfaces, their gradients, and the matrix elements coupling the surfaces. The need for the couplings can be alleviated by employing the time derivatives of the wave functions, which can be evaluated from overlaps of electronic wave functions at successive timesteps. However, evaluation of overlap integrals is still expensive for large systems. In addition, for electronic structure methods for which the wave functions or the coupling matrix elements are not available, nonadiabatic dynamics algorithms become inapplicable. In this work, building on recent work by Baeck and An, we propose new nonadiabatic dynamics algorithms that only require adiabatic potential energies and their gradients. The new methods are named curvaturedriven coherent switching with decay of mixing ( $\kappa \mathrm{CSDM})$ and curvature-driven trajectory surface hopping ( $\kappa \mathrm{TSH})$. We show how powerful these new methods are in terms of computer time and good agreement with methods employing nonadiabatic coupling vectors computed in conventional ways. The lowering of the computational cost will allow longer nonadiabatic trajectories and greater ensemble averaging to be affordable, and the ability to calculate the dynamics without electronic structure coupling matrix elements extends the dynamics capability to new classes of electronic structure methods. 


\section{Introduction}

Simulating the dynamics of processes involving electronically excited molecules requires potential energy surfaces, their gradients, the electronic matrix elements controlling their coupling, and a nonadiabatic dynamics algorithm. ${ }^{1,2,3,4,5,6,7,8,9,10,11,12,13,14,15,16,17,18,19}$ Recent advances on these topics have enabled quantitative accurate simulations for medium-size molecules and qualitatively accurate simulations for nano-sized molecules or clusters. $^{20,21,22,23,24,25,26,27,28,29,30,31}$ The electronic structure must be treated quantum mechanically, and for simulating all but the smallest systems, one uses a semiclassical treatment of nuclear motion. The present article is concerned with direct dynamics, where, instead of requiring parametrized analytic functions for the energies and couplings, all required energies, forces, and couplings for each geometry that is important for evaluating dynamical properties are obtained directly from electronic structure calculations when they are needed in the dynamics calculation. Electronic structure calculations directly yield electronically adiabatic wave functions, $\phi_{I}$, and the coupling in the semiclassical calculations in the adiabatic representation is provided by the nuclear momentum operator, which involves the matrix elements of the gradient with respect to nuclear coordinates $\mathbf{R}:^{1,2,32}$

$$
\mathbf{d}_{I J}=\left\langle\phi_{I}|\partial / \partial \mathbf{R}| \phi_{J}\right\rangle
$$

where $\langle\ldots|\ldots| \ldots\rangle$ denotes an electronic matrix element. The matrix element in eq 1 is usually called the nonadiabatic coupling (NAC). It is a $3 N$-dimensional vector where $N$ is the number of atoms. The present article is concerned with an efficient approximation to the NAC.

In semiclassical methods, the electronic wave functions may also be considered to be functions of time $(t)$ because

$$
\phi_{I}=\phi_{I}(\mathbf{r} ; \mathbf{R}(t))
$$

where $\boldsymbol{r}$ denotes the electronic coordinates, and $\mathbf{R}(t)$ is the trajectory of nuclear motion. A popular semiclassical method is trajectory surface hopping (TSH). ${ }^{1,2,9,33,34,35,36,37,38}$ In TSH one only requires the NAC in the direction of the current velocity, $\dot{\mathbf{R}}$, because the semiclassical equations only require the time derivative coupling (TDC):

$$
\sigma_{I J}=\left\langle\phi_{I}|d / d t| \phi_{J}\right\rangle=\left\langle\phi_{I}|\partial / \partial \mathbf{R}| \phi_{J}\right\rangle \cdot \dot{\mathbf{R}}
$$


Employing a direct calculation of the TDC is more efficient than calculating the NAC, and it can be shown to yield more numerically accurate integration of the TSH electronic equations of motion than direct use of the NAC, especially for situations of trivial crossings. ${ }^{39,40,41,42}$

More complete semiclassical methods, like semiclassical Ehrenfest ${ }^{43,44,45,46,47}$ (SE) dynamics and coherent switching with decay of mixing 4,48,49,50,51,52 (CSDM) dynamics, require all components of the NAC. Because the evaluation of the time derivative is more efficient than the evaluation of a NAC, we have recently formulated an effective $\mathrm{NAC}^{53}$ that can be computed from the TDC and that can be used to carry out SE and CSDM dynamics with only the time derivative (without requiring a full NAC calculation). We also reported test calculations showing that using the effective NAC instead of the actual NAC does not cause a large error. These variants of SE and CSDM are called tSE and tCSDM, where " $\mathrm{t}$ " denotes time derivative. The TDC needed for such calculations can be approximated in various ways by overlaps of electronic wave functions at the current and previous time step of the trajectory. ${ }^{38,53,54}$ Therefore, TSH, tSE, and tCSDM can be all be calculated efficiently without computing NACs from electronic structure software.

Not only is the effective NAC preferred for its convenience and efficiency, but also it may be preferred for new or high levels of electronic structure where NACs are not yet present in available software. For nonvariational methods, like configuration interaction without statespecific self-consistent orbitals, ${ }^{55}$ coupled cluster theory, ${ }^{56,57}$ or multi-configuration pair-density functional theory, ${ }^{58,59,60}$ the NAC can be computed by linear response with a Lagrangian formulation, ${ }^{61,62,63}$ but such calculations can be inconvenient and are often unavailable. Another class of electronic structure methods where NACs may be unavailable is provided by the recently popular machine-learning-based energy calculations. ${ }^{64,65,66,67}$ For these reasons, it would be convenient to have approximate NACs that can be evaluated straightforwardly from energies and energetic derivatives. We therefore ask, can we approximate the TDC and effective NAC from energy information, and does this provide enough accuracy for efficient propagation of dynamics? The present paper addresses this question.

We will introduce new nonadiabatic dynamics algorithms that utilize curvature of the energy gap to approximate the TDC and the effective $3 N$-dimensional NAC, and we will show how they can be used to develop new variants of tSE and tCSDM that we shall call curvaturedriven semiclassical Ehrenfest ( $\mathrm{KSE}$ ) and curvature-driven coherent switching with decay of 
mixing $(\kappa \mathrm{CSDM})$. Both $\kappa \mathrm{SE}$ and $\kappa \mathrm{CSDM}$ require only energy and gradient information from electronic structure theory. The curvature-approximated TDC introduced in the current work is an extension of the approximate NAC previously developed for one-dimensional systems by Baeck and An $(\mathrm{BA})^{68}$

$$
\mathbf{d}_{I J}^{\mathrm{BA}}=\left\langle\phi_{I}|d / d q| \phi_{J}\right\rangle \cong \frac{1}{2}\left[\frac{d^{2}\left(V_{I}-V_{J}\right)}{d q^{2}} \frac{1}{V_{I}-V_{J}}\right]^{1 / 2}
$$

where $V_{I}$ and $V_{J}$ are adiabatic potential energies, and $q$ is a one-dimensional nuclear coordinate.

The CSDM algorithm is based on SE, but in CSDM the SE algorithm is augmented by nonMarkovian decoherence and stochastic pointer-state switches. CSDM inherits the self-consistent potential advantage of SE and SE's robustness to on the choice of electronic wave function representation, and it shares with TSH the stochastic switching to physical final states, but CSDM does not have TSH's discontinuous nuclear momenta or its frustrated hops problem. Perhaps most importantly of all, CSDM balances coherence in strong interaction regions with decoherence between them in a fashion consistent with the Liouville-von Neumann equation. CSDM was initially applied to processes conserving electron spin, where it showed good agreement with accurate quantum dynamics, ${ }^{49,51}$ and the recent implementation of CSDM in the SHARC software package ${ }^{19,52,69,70}$ has enabled it to conveniently describe intersystem crossing processes. ${ }^{71}$

\section{Theory}

Since eq 4 is the one-dimensional derivative with respect to a coordinate in the direction of motion, we generalize it to the following one-dimensional derivative with respect to time:

$$
\kappa \mathrm{TDC} \equiv \sigma_{I J}^{\kappa}=\left\langle\phi_{I}|d / d t| \phi_{J}\right\rangle \cong \frac{1}{2}\left[\frac{d^{2}\left(V_{I}-V_{J}\right)}{d t^{2}} \frac{1}{V_{I}-V_{J}}\right]^{1 / 2}, \quad \text { for } J>I
$$

where $\left(V_{I}-V_{J}\right)$ is the local gap between adiabatic potential surfaces, and we use $\kappa$ as a prefix and as a superscript to denote approximation based on the curvature of the gaps. Since the NAC is skew-Hermitian, we also have

$$
\sigma_{J I}^{\kappa}=-\sigma_{I J}^{\kappa}
$$


Employing eqs 5 and 6 for the TDC is the central feature of $\kappa$ SE and $\kappa C S D M$. This approximation can also be employed in TSH, yielding $\kappa \mathrm{TSH}$. We have implemented $\kappa \mathrm{SE}$, $\kappa \mathrm{CSDM}$, and $\kappa \mathrm{TSH}$ in a new version of SHARC-MN, which is available at our cost at our software distribution site. ${ }^{72}$ Next we give (i) the details of the how we calculate the TDC numerically in SHARC-MN and (ii) the remaining details necessary to completely specify $\kappa \mathrm{SE}$ and $\kappa$ CSDM, which - as stated above - require an effective NAC as well as the TDC.

кTDC. The curvature-approximated TDC is given in general by eqs 5 and 6 . However, there are some details that need to be clarified.

First, when the radicand is negative, we simply set it to zero. This is justified as follows. The reason why a formula like eq 4 or q 5 works is best appreciated by considering the nature of two potential curves near a locally avoided crossing. In a typical case the lower curve is concave, and the upper curve is convex, so the radicand is positive. Therefore, a situation where the radicand is negative is expected to occur only far from conical intersections where the NAC is small, so this should not cause a large error.

Second, we note that $\sigma_{I J}^{\kappa}$ can be re-written as,

$$
\sigma_{I J}^{\kappa}=\frac{1}{2}\left[\frac{d\left(\frac{\partial V_{I}}{\partial \mathbf{R}} \cdot \dot{\mathbf{R}}-\frac{\partial V_{J}}{\partial \mathbf{R}} \cdot \dot{\mathbf{R}}\right)}{d t} \frac{1}{V_{I}-V_{J}}\right]^{1 / 2}
$$

In the practical SHARC-MN implementation, this is computed from backward finite differences as follows:

$$
\sigma_{J I}^{\kappa}(t+\Delta t) \approx \frac{1}{2}\left[\frac{\Delta \dot{V}_{J I}(t+\Delta t)-\Delta \dot{V}_{J I}(t)}{\Delta t} \frac{1}{V_{J}-V_{I}}\right]^{1 / 2} \quad \text { for } J>I
$$

where

$$
\Delta \dot{V}_{J I}(t)=\frac{\partial V_{J}(t)}{\partial \mathbf{R}} \cdot \dot{\mathbf{R}}(t)-\frac{\partial V_{I}(t)}{\partial \mathbf{R}} \cdot \dot{\mathbf{R}}(t)
$$

SHARC employs velocity-Verlet algorithm, ${ }^{73}$ in which the velocity at time step $t+\Delta t$ is evaluated after the nuclear force at $t+\Delta t$ is computed.

For both SE and CSDM, the nuclear forces are not decidable until one has determined the electronic coefficients. And therefore, $\mathbf{R}(t+\Delta t)$, which is needed when eq 9 is substituted into 
eq 8 , is not available for evaluating $\sigma_{J I}^{\kappa}(t+\Delta t)$ when one uses the velocity-Verlet algorithm. Hence, in the current implementation, $\dot{\mathbf{R}}(t+\Delta t)$ is approximated by a forward propagation,

$$
\dot{\mathbf{R}}(t+\Delta t) \approx \dot{\mathbf{R}}(t)+\mathbf{a}(t) \Delta t
$$

where $\mathbf{a}(t)$ is acceleration vector at time $t$.

For TSH, when uses the velocity-Verlet algorithm, one does have $\dot{\mathbf{R}}(t+\Delta t)$ when evaluating the TDC. However, when a hop happens, the velocity needs to be adjusted to enforce energy conservation. Therefore, to make a consistent approximation over the whole trajectory, we employ eq 10 to evaluate $\dot{\mathbf{R}}(t+\Delta t)$ for $\kappa \mathrm{TSH}$ as well.

кTSH. In the curvature-driven TSH method, $\kappa \mathrm{TSH}$, the electronic equation of motion employs $\kappa$ TDC computed by eqs 5 and 6 instead of directly computing of the time derivative coupling either by eq 3 or from overlaps of electronic wave functions at successive steps. Furthermore, in $\kappa \mathrm{TSH}$, the velocity rescaling direction after a successful hop is the difference gradient vector (eq 6 in ref 74).

KSE. The SE method in an adiabatic basis involves an expansion of the electronic wave function in an $N$-state basis:

$$
\varphi^{\mathrm{elec}}(\mathbf{r} ; \mathbf{R}(t))=\sum_{J=1}^{N} c_{J}(t) \phi_{J}(\mathbf{r} ; \mathbf{R}(t))
$$

where $c_{J}$ is the coefficient of adiabatic electronic state $\phi_{J}$. Inserting eq 11 into the time dependent electronic Schrödinger equation, using eqs 5 and 6 for the TDC, and performing some straightforward calculus gives the electronic equation of motion (EOM) for $\kappa \mathrm{SE}$ :

$$
\frac{d c_{I}}{d t}=-\frac{i}{\hbar}\left(c_{I}(t) V_{I}-i \hbar \sum_{J \neq I}^{N} c_{I}(t) \sigma_{I J}^{\kappa}\right)
$$

The nuclear EOM of the regular SE method is

$$
\dot{\mathbf{P}}(t)=-\sum_{I} \rho_{I I} \frac{\partial V_{I}}{\partial \mathbf{R}}+\sum_{I} \sum_{J \neq I} \operatorname{Re}\left(\rho_{I J}\right)\left(V_{I}-V_{J}\right) \mathbf{d}_{I J}
$$

where $\mathbf{P}$ is the momentum conjugate to $\mathbf{R}$, and

$$
\rho_{I J}=c_{I} c_{J}^{*}
$$


is an element of the electronic density matrix. For the tSE, method, we re-derived the nuclear EOM such that it depends on an effective NAC:

$$
\dot{\mathbf{P}}=-\sum_{I} \rho_{I I} \frac{\partial V_{I}}{\partial \mathbf{R}}+\sum_{I} \sum_{J \neq I}\left[\frac{\operatorname{Re}\left(\rho_{I J}\right)\left(V_{I}-V_{J}\right) \sigma_{I J}}{\mathbf{v} \cdot \mathbf{G}_{I J}}\right] \mathbf{G}_{I J}
$$

where $\mathbf{G}_{I J}$ is the effective NAC defined by the difference gradient vector $\mathbf{g}_{I J}$, the velocity vector $\dot{\mathbf{R}}$, and the TDC:

$$
\begin{gathered}
\mathbf{G}_{I J}=\mathbf{g}_{I J}+\alpha \dot{\mathbf{R}} \\
\mathbf{g}_{I J}=\frac{\partial V_{I}}{\partial \mathbf{R}}-\frac{\partial V_{J}}{\partial \mathbf{R}} \\
\alpha=\frac{\sigma_{I J}-\dot{\mathbf{R}} \cdot \mathbf{g}_{I J}}{\dot{\mathbf{R}} \cdot \dot{\mathbf{R}}}
\end{gathered}
$$

We justified calling $\mathbf{G}_{I J}$ an effective NAC because it satisfies

$$
\dot{\mathbf{R}} \cdot \mathbf{G}_{I J}=\sigma_{I J}
$$

We now replace $\sigma_{I J}$ in eq 18 by $\sigma_{I J}^{\kappa}$, such that eqs $17-19$ yield a new effective NAC, which will be called the curvature-approximated effective NAC and denoted by $\mathbf{G}_{I J}^{\kappa}$.

As before ${ }^{75}$ it is necessary to project out the rotational and translational components of the effective NAC in order to conserve the total angular momentum and the position of the center of mass. This yields ${ }^{75}$

$$
\mathbf{G}_{I J}^{\mathrm{\kappa} \mathbf{Q}}=(\mathbf{1}-\mathbf{Q}) \mathbf{G}_{I J}^{\kappa}
$$

where $\mathbf{1}$ and $\mathbf{Q}$ are the identity matrix and a projection operator.75 The operation in eq 20 removes unphysical translational and rotational components of $\mathbf{G}_{I J}^{\kappa}$. Therefore the nuclear EOM of $\kappa \mathrm{SE}$ becomes:

$$
\dot{\mathbf{P}}=-\sum_{I} \rho_{I I} \frac{\partial V_{I}}{\partial \mathbf{R}}+\sum_{I} \sum_{J \neq I}\left[\frac{\operatorname{Re}\left(\rho_{I J}\right)\left(V_{I}-V_{J}\right) \sigma_{I J}^{\kappa}}{\mathbf{v} \cdot \mathbf{G}_{I J}^{\mathrm{\kappa} \mathbf{Q}}}\right] \mathbf{G}_{I J}^{\mathrm{\kappa} \mathbf{Q}}
$$

Equations 12 and 21 define the electronic and nuclear EOM for $\kappa \mathrm{SE}$.

кCSDM. Adding decoherence to $\kappa \mathrm{SE}$ in the same that it was added to tSE to get $\mathrm{TCSDM}^{53}$ yields the following electronic and nuclear EOMs for $\kappa$ CSDM:

$$
\left[\dot{c}_{I}\right]_{\mathrm{KCSDM}}=\left[\dot{c}_{I}\right]_{\mathrm{KSE}}+\left[\dot{c}_{I}\right]_{\mathrm{DM}}
$$




$$
[\dot{\mathbf{P}}]_{\mathrm{KCSDM}}=[\dot{\mathbf{P}}]_{\mathrm{KSE}}+[\dot{\mathbf{P}}]_{\mathrm{DM}}
$$

where $\left[\dot{c}_{I}\right]_{\mathrm{KSE}}$ and $[\dot{\mathbf{P}}]_{\mathrm{KSE}}$ denote the electronic and nuclear equation of motion from $\kappa \mathrm{SE}$, as given in eqs 12 and 21, and where the additional terms are decay-of-mixing (DM) terms, which are explained in detail elsewhere. ${ }^{48,53}$ In tCSDM decoherence contribution to the change in the nuclear momentum is ${ }^{53}$

$$
[\dot{\mathbf{P}}(t)]_{\mathrm{DM}}=\sum_{I \neq K}^{N} \frac{\rho_{I I}(t)}{\tau_{I K}} \frac{\left(V_{I}-V_{K}\right)}{\left(\mathbf{s}_{I K} \cdot \mathbf{v}\right)} \mathbf{s}_{I K}
$$

in which $\tau_{I K}$ is the decoherence time, $K$ is the pointer state, and $\mathbf{s}_{I K}$ is the decoherence vector for states $I$ and $K$ given by

$$
\mathbf{s}_{I K}=\operatorname{Re}\left(\frac{a_{0} \mathbf{P}_{\mathrm{vib}} \cdot \mathbf{G}_{I J}^{\mathbf{Q}}}{\left|\mathbf{G}_{I J}^{\mathbf{Q}}\right|} \mathbf{G}_{I J}^{\mathbf{Q}}\right)+\mathbf{P}_{\mathrm{vib}}
$$

where $\mathbf{P}_{\mathrm{vib}}$ is the internal vibrational momentum, and $a_{0} \equiv 1$ bohr.

In CSDM, tCSDM, and $\mathrm{KCSDM}$, we propagate two density matrices; the true density matrix is propagated by equations 22 and 14, and another density matrix, called the coherent density matrix, is propagated coherently (i.e., by eqs 12 and 14) and is used to control the switching of the pointer state. Although the coherent density matrix is in general different from true density along the trajectory, it is re-initialized to true density for every complete passage of a strong interaction region.

Beyond the changes already discussed for $\mathrm{\kappa SE}$, two additional changes are required to obtain $\kappa$ CSDM:

In tCSDM, the complete passage of a strong interaction region is defined by local minima of

$$
D_{K}(t)=\sum_{I \neq K}^{N}\left|\sigma_{I K}\right|
$$

In $\kappa \mathrm{CSDM}$, we replace this by

$$
D_{K}(t)=\sum_{I \neq K}^{N}\left|\sigma_{I K}^{\kappa}\right|
$$

The decoherence direction of eq 25 is replaced by 


$$
\mathbf{s}_{I K}=\frac{a_{0} \mathbf{P}_{\mathrm{vib}} \cdot \mathbf{G}_{I K}^{\mathrm{\kappa} \mathbf{Q}}}{\left|\mathbf{G}_{I K}^{\mathrm{\kappa} \mathbf{Q}}\right|} \mathbf{G}_{I K}^{\mathrm{K} \mathbf{Q}}+\mathbf{P}_{\mathrm{vib}}
$$

\section{Computational details}

We implemented $\kappa$ SE, $\kappa \mathrm{CSDM}$, and $\kappa \mathrm{TSH}$ in SHARC-MN,${ }^{72}$ and we tested $\kappa \mathrm{CSDM}$ against the previously published CSDM and ICSDM methods. We used nonadiabatic dynamics of the ethylene molecule as our test example. The Molpro software package ${ }^{76}$ was interfaced to SHARC-MN to perform direct dynamics.

Ground-state geometry optimization of ethylene together with vibrational analysis were performed with MP2/6-31G**,77,78 and the initial geometries and velocities of the trajectories were randomly sampled from the ground-vibrational-state Wigner distribution. Before propagating the EOMs, the molecule was raised vertically to the first excited state $\left(\mathrm{S}_{1}\right)$.

We performed tests of $\kappa \mathrm{CSDM}$ and $\kappa \mathrm{TSH}$. We do not test $\kappa \mathrm{SE}$ because we use SE and $\kappa \mathrm{SE}$ simply as base methods upon which to build CSDM and $\kappa$ CSDM - not preferred choices for practical simulations.

The potential energy surfaces and gradients for ethylene direct dynamics were calculated by state-averaged complete-active-space self-consistent-field theory (SA-CASSCF) ${ }^{79,80}$ by averaging over 3 states with a $(2,2)$ activated space (two active electrons in two active orbitals) and the $6-31 \mathrm{G}^{* *}$ basis set.

We ran 300 trajectories for $\mathrm{KCSDM}$ and 150 trajectories each for CSDM and tCSDM. We ran these trajectories with a 0.1 fs nuclear dynamics time step time step and 200 substeps for the integration of the electronic EOM. The trajectories were terminated after $250 \mathrm{fs}$ or earlier when the ground state population remains larger than 0.98 for $20 \mathrm{fs}$. (In order to include the earlytermination trajectories in the time-dependent plots of populations and state energies, we keep the values of the population and state energies constant from the value at termination out to 250 fs.)

We also ran trajectory surface hopping with the energy-based decoherence correction ${ }^{81}$ (TSH-EDC). We ran 200 TSH-EDC trajectories - 100 using the time derivative calculated as an overlap and 100 using the scalar product of the NAC and the velocity. With converged ensemble averaging and converged step sizes and if one sued the same velocity rescaling at hops, one would get the same result by these two methods so in that sense it is legitimate to combine their results; however, we ran these trajectories with both algorithms so we could compare the timing. 
When we need to distinguish thee two algorithms, we label them as TSH-EDC(TDC) and TSHEDC(NAC).

Finally, we ran 100 trajectories for $\kappa \mathrm{TSH}-\mathrm{EDC}$. The decoherence correction is the same as in TSH-EDC. The velocity component in the direction of the gradient difference is rescaled to adjust the kinetic energy after a successful surface hop for $\kappa$ TSH-EDC and TSH-EDC(TDC), but for TSH-EDC(NAC), the direction of the projected NAC is used to adjust kinetic energy after a successful surface hop.

\section{Results}

The present results for $\kappa$ CSDM are compared to our previous results ${ }^{53}$ for CSDM and tCSDM. To assess the excited-state lifetime, we employ ensemble-averaged population analysis using the following definition of the probability of being in state $I$ :

$$
\left\langle\rho_{I I}\right\rangle=\sum_{\xi=1}^{N_{\text {traj }}} \frac{\rho_{I I}^{\xi}}{N_{\text {traj }}}
$$

where $\xi$ is the index of trajectory, $N_{\text {traj }}$ is the total number of trajectories, and the probability is evaluated when the trajectory is terminated. The ensemble-averaged populations in the ground and excited states are shown as functions of time in Fig 1. Alternatively, we can define the population of a certain state $I$ as,

$$
\left\langle\rho_{I I}\right\rangle=\frac{N_{\text {traj }}^{I}}{N_{\text {traj }}}
$$

where $N_{\text {traj }}^{I}$ is the number of trajectories for which the pointer state is $I$ when the trajectory is terminated. This population is denoted as the pointer-state population. The pointer-state population for $\kappa$ CSDM, tCSDM, and CSDM is shown in Fig. S1 in Supporting Information.

The ensemble-averaged potential energies for $\mathrm{S}_{0}, \mathrm{~S}_{1}, \mathrm{~S}_{2}$ and the effective PES (eq 8 of ref 48) are shown in Fig 2. The effective PES (denoted as $V_{\text {eff) }}$ is gradually changing from $V_{1}$ to $V_{0}$ around $50 \mathrm{fs}$ which is consistent with the time region where population transfers from $\mathrm{S}_{1}$ to $\mathrm{S}_{0}$. The half-life $\left(\tau_{1 / 2}\right)$ obtained from the simulations for decay of the $\mathrm{S}_{1}$ excited state are 60,52 , and 51 fs for $\kappa$ CSDM, tCSDM, CSDM, respectively, which shows very good agreement among the three methods; in fact, the difference is of the same order as the statistical uncertainty in the halflife due to the finite amount of Monte Carlo ensemble averaging with 150-300 trajectories). 
To assess the accuracy of curvature-approximated TDC and the curvature-approximated effective NAC, we randomly picked three $\kappa \mathrm{CSDM}$ trajectories and performed NAC calculation at each geometry along the trajectory. The norm of ab initio NAC $\mathbf{d}_{I J}$ and projected curvatureapproximated effective NAC $\mathbf{G}_{I J}^{\mathrm{kQ}}$ for $\mathrm{S}_{0}$ and $\mathrm{S}_{1}$ are shown in Fig. 3. For comparison, this figure also shows $V_{0}$ and $V_{1}$ along the $\kappa \mathrm{CSDM}$ trajectory. It is clear that $\mathbf{d}_{12}$ and $\mathbf{G}_{12}^{\mathrm{K} \mathbf{Q}}$ spike at the same position, which is where $V_{0}$ and $V_{1}$ get close. However, we also observe a trend that $\mathbf{G}_{12}^{\mathrm{K} \mathbf{Q}}$ seems to be relatively smaller than $\mathbf{d}_{12}$, and that may explain why population transfer from $\mathrm{S}_{1}$ to $\mathrm{S}_{0}$ seems to be slightly faster in TCSDM and CSDM than in $\mathrm{KCSDM}$; however, it is not clear if the difference is statistically significant. The bottom line is that the curvature-driven method is in good agreement with tCSDM and CSDM.

The computational cost ratio for $\kappa$ CSDM, tCSDM, and CSDM is roughly $1: 3.5: 4.5$. These ratios show a significant speedup for $\kappa \mathrm{CSDM}$, which occurs because no overlap integrals or nonadiabatic coupling vectors are computed from electronic structure software.

Next, we discuss $\kappa \mathrm{TSH}$. The $\mathrm{S}_{0}, \mathrm{~S}_{1}$, and $\mathrm{S}_{2}$ populations (computed by eq 29) as a function of time for $\kappa$ TSH-EDC, TSH-EDC(TDC), and TSH-EDC(NAC) are shown in Fig 4. The half-lives $\left(\tau_{1 / 2}\right)$ for the $S_{1}$ excited state obtained from the simulations are 63 and 62 fs for $\kappa$ TSH-EDC and TSH-ED, respectively. These results indicate that $\mathrm{\kappa TSH}-\mathrm{EDC}$ is a very good approximation to TSH-EDC. The ensemble averaged potential energies as a function of time for $\kappa$ TSH-EDC and TSH-EDC are shown in Fig 5.

The pointer state populations as a function of time for KTSH-EDC and TSH-EDC are shown in Fig. S2 in Supporting Information.

The computation time ratios for KTSH-EDC, TSH-EDC(TDC), TSH-EDC(NAC), and $\kappa C S D M$ are $0.7: 0.8: 1.6: 1.0$. This shows that the speedup of $\kappa$ TSH-EDC relative to TSH$\mathrm{EDC}(\mathrm{NAC})$ is more than a factor of two even for this small molecule (as the molecule simulated gets larger, this timing savings is expected to become more significant; it could even be dramatic). However, $\kappa \mathrm{TSH}$ is only $30 \%$ faster than the more accurate $\kappa \mathrm{CSDM}$ method. Furthermore, these timings show that $\mathrm{KTDC}$ drops the cost of the mean-field, dual-density-matrix $\kappa C S D M$ algorithm below that of TSH-EDC(NAC). 


\section{Concluding Remarks}

In this work, we proposed a curvature-approximated time-derivative coupling formula, and we showed how to use this formula to obtain curvature-driven approximations to trajectory surface hopping, to the semiclassical Ehrenfest method, and to the coherent switching with decay of mixing method for nonadiabatic dynamics. The curvature-driven methods are called $\kappa \mathrm{TSH}$, $\kappa \mathrm{SE}$, and $\kappa \mathrm{CSDM}$. We demonstrated for ethylene that nonadiabatic dynamics with $\kappa \mathrm{CSDM}$ and $\kappa T S H-E D C$ gives results close to those given by CSDM and TSH-EDC. The curvature-driven methods do not require the calculations of nonadiabatic coupling vectors (NACs) or time derivatives of the wave function. Thus they may be used to speed up calculations as compared to using electronic-structure nonadiabatic couplings (NACs). For ethylene we found a speedup of $\kappa \mathrm{CSDM}$ by a factor of 4.5 relative to CSDM and a speedup of $20-30 \%$ for $\kappa$ TSH-EDC relative to TSH-EDC. Such speedup may enable longer-time trajectories and/or better ensemble averaging. Even more promising is that curvature-driven couplings can be used for direct dynamics calculations of electronically nonadiabatic processes with electronic structure methods for which NACs or time derivatives are not available.

\section{Software Availability}

The new methods described in this article are available (free) in $S H A R C-M N-v 1.1,{ }^{72}$ which is based on SHARC-v2.1. ${ }^{82}$

\section{- ASSOCIATED CONTENT}

\section{S Supporting Information}

The Supporting Information is available free of charge on the ACS Publications website at DOI: $10.1021 /$

Sample input files for SHARC-MN

\section{AUTHOR INFORMATION \\ Corresponding Authors}

Donald G. Truhlar - Department of Chemistry and Supercomputing Institute, University of Minnesota, Minneapolis, Minnesota 55455-0431, United States; orcid.org/0000- 0002-77427294; Email: truhlar@umn.edu

Xiye Chen - School of Energy Science and Engineering, Harbin Institute of Technology, Harbin 150001, People's Republic of China; School of Chemistry and Chemical Engineering, Harbin Institute of Technology, Harbin 150001, People's Republic of China; orcid.org/0000-0003-36888032; Email: chenxiye@hit.edu.cn 


\section{Authors}

Yinan Shu - Department of Chemistry and Supercomputing Institute, University of Minnesota, Minneapolis, Minnesota 55455-0431, United States; orcid.org/0000-0002-8371-0221

Linyao Zhang - School of Energy Science and Engineering, Harbin Institute of Technology, Harbin 150001, People's Republic of China; School of Chemistry and Chemical Engineering, Harbin Institute of Technology, Harbin 150001, People's Republic of China; orcid.org/00000003-1848-8860

Shaozeng Sun - School of Energy Science and Engineering, Harbin Institute of Technology, Harbin 150001, People's Republic of China; orcid.org/0000-0003-2793-5925

Yudong Huang - School of Chemistry and Chemical Engineering, Harbin Institute of Technology, Harbin 150001, People's Republic of China; orcid.org/0000-0002-9998-557X

\section{Authors Contributions}

$\S$ Y.S. and L.Z.: These authors contributed equally.

\section{Notes}

The authors declare no competing financial interest.

\section{ACKNOWLEDGMENTS}

This work was supported in part by the U.S. Department of Energy, Office of Science, Office of Basic Energy Sciences under Award DE-SC0015997 and by the National Natural Science Foundation of China under grant No. 51536002. 


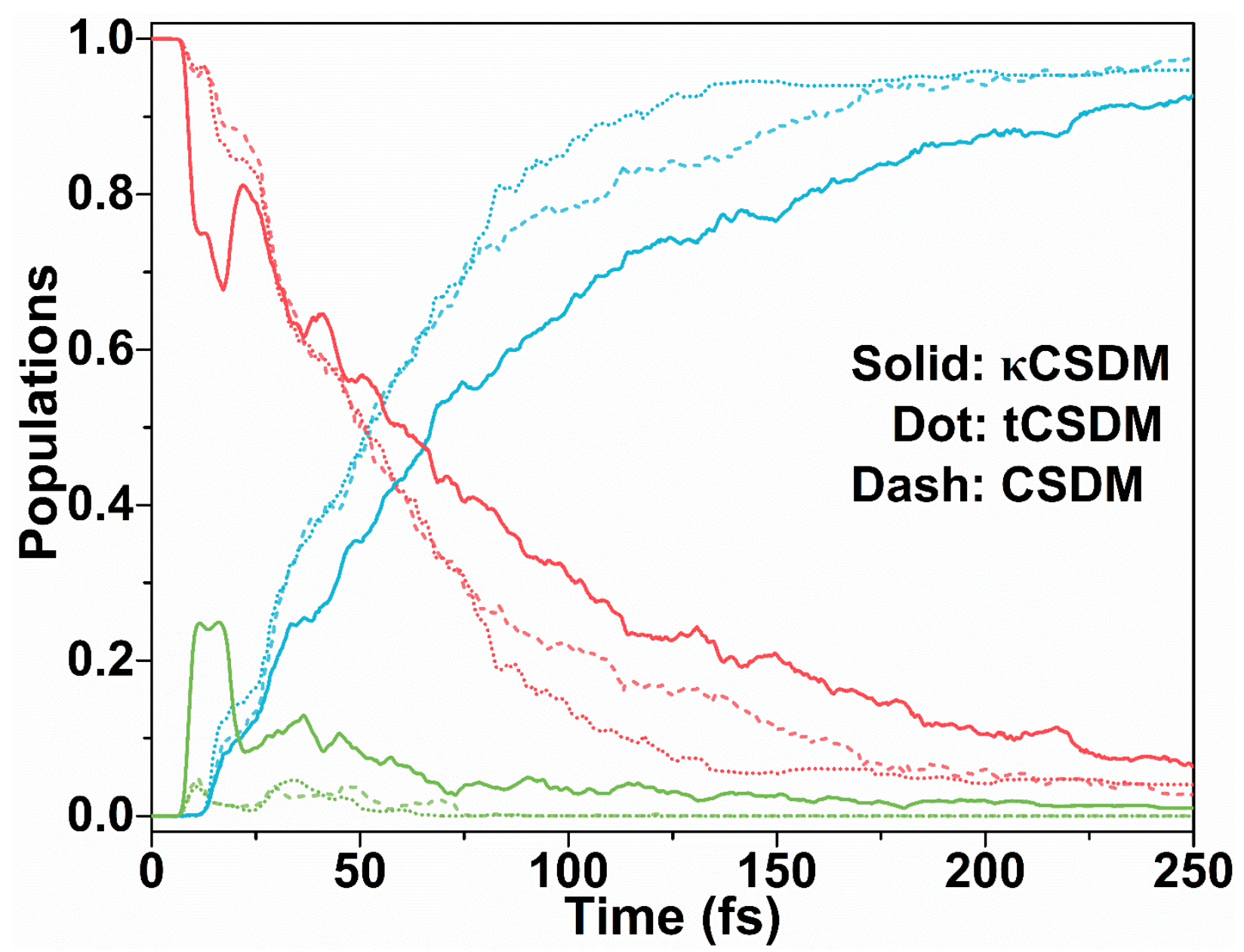

Figure 1. $\mathrm{S}_{0}$ (red), $\mathrm{S}_{1}$ (blue), and $\mathrm{S}_{2}$ (green) populations as functions of time for $\kappa \mathrm{CSDM}$ (solid), tCSDM (dotted) and CSDM (dashed). The curves represent averages over the ensemble of trajectories for the given method. 


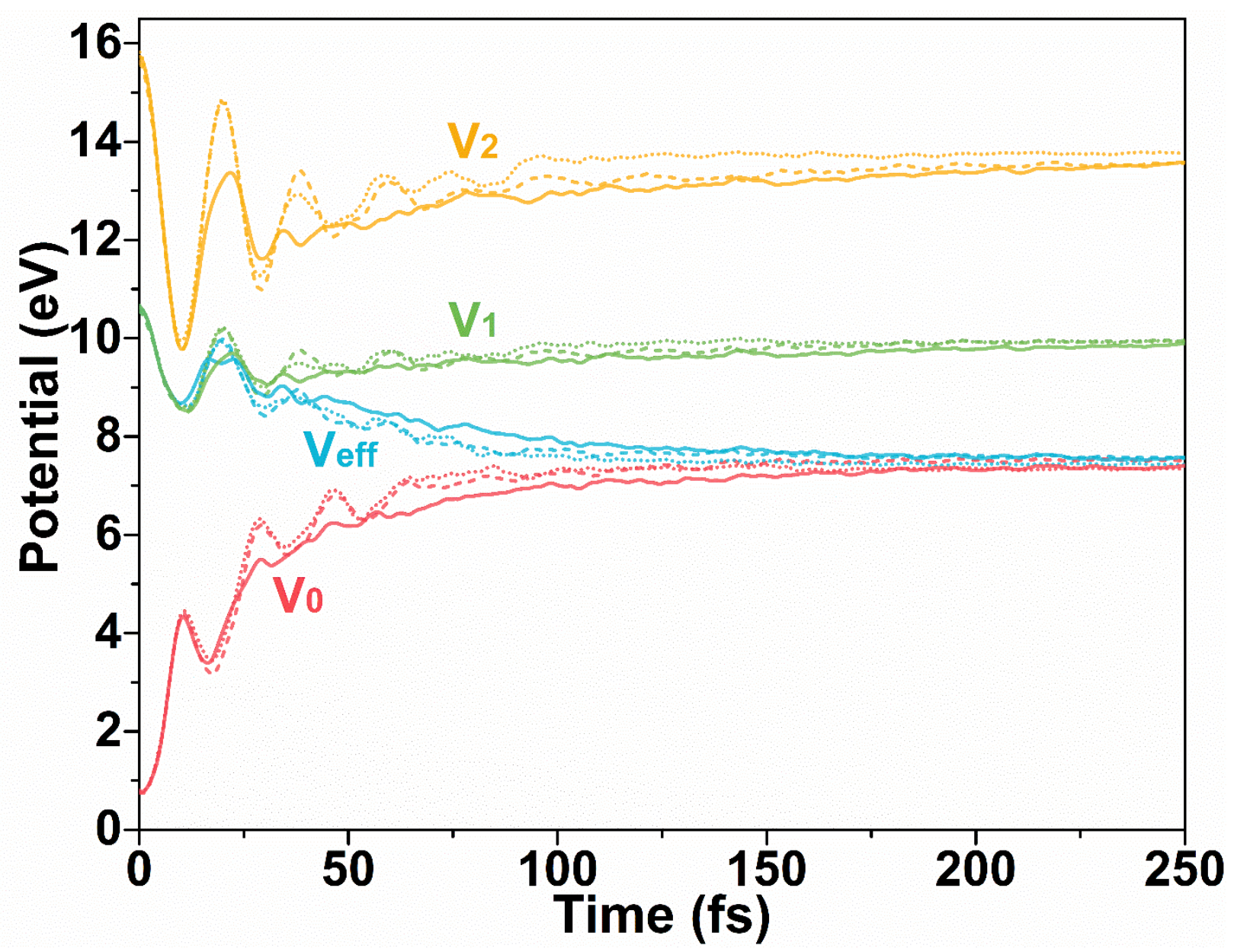

Figure 2. $\mathrm{S}_{0}, \mathrm{~S}_{1}, \mathrm{~S}_{2}$ and mean-field PESs denoted as $\mathrm{V}_{0}$ (red), $\mathrm{V}_{1}$ (green), $\mathrm{V}_{2}$ (yellow), and $\mathrm{V}_{\text {eff }}$ (blue) as functions of time for KCSDM (solid), tCSDM (dotted) and CSDM (dashed). The curves represent averages over the ensemble of trajectories for the given method. 


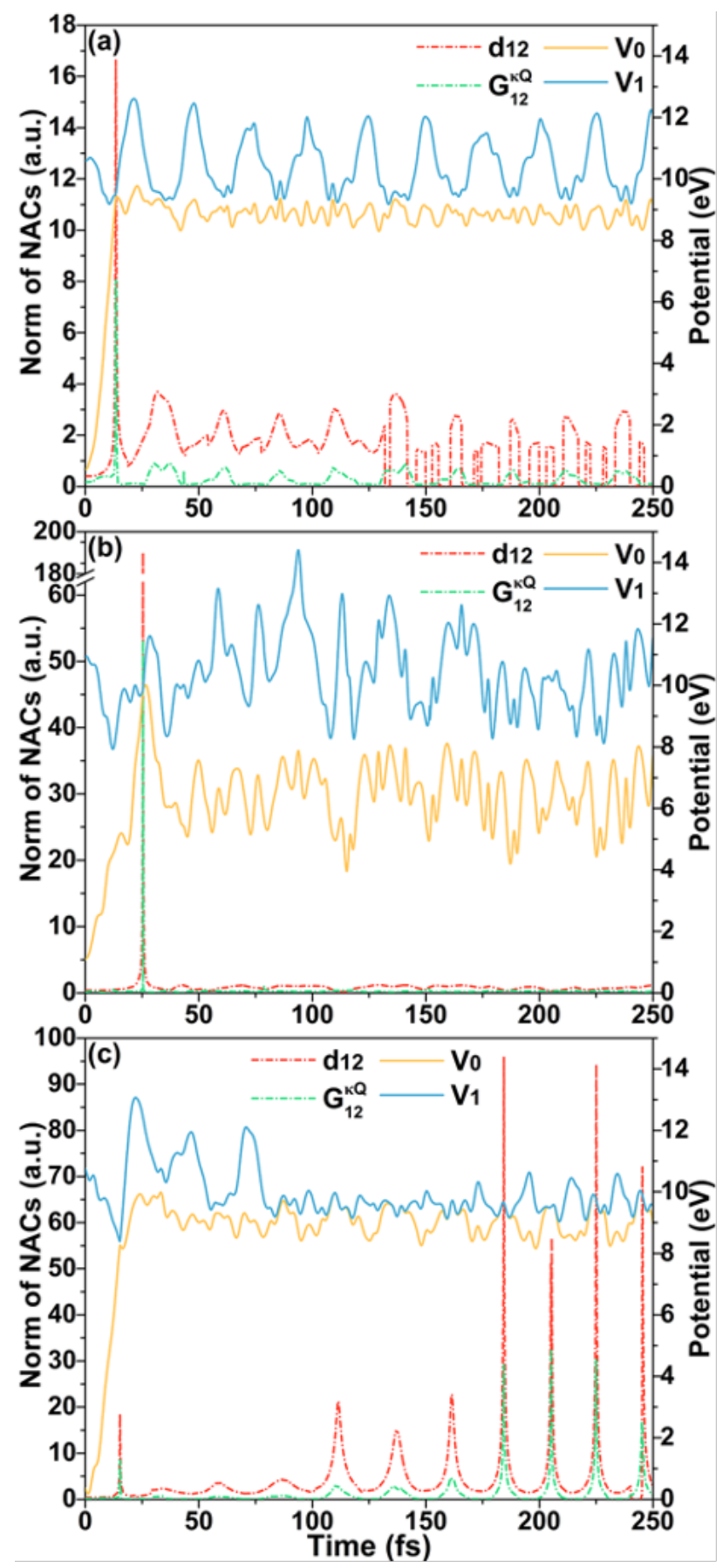

Figure 3. The norm of $a b$ initio NAC $\mathbf{d}_{12}$ and the magnitude of the projected curvatureapproximated effective NAC $\mathbf{G}_{12}^{\kappa \mathbf{Q}}$ along three randomly selected $\kappa \mathrm{CSDM}$ trajectories are shown in red and green respectively. We also show the adiabatic potentials $\mathrm{V}_{0}$ (yellow) and $\mathrm{V}_{1}$ (blue) for comparison. Note that states $\mathrm{S}_{0}$ and $\mathrm{S}_{1}$ are numbered 1 and 2 in the row and column indices of the matrix elements. 


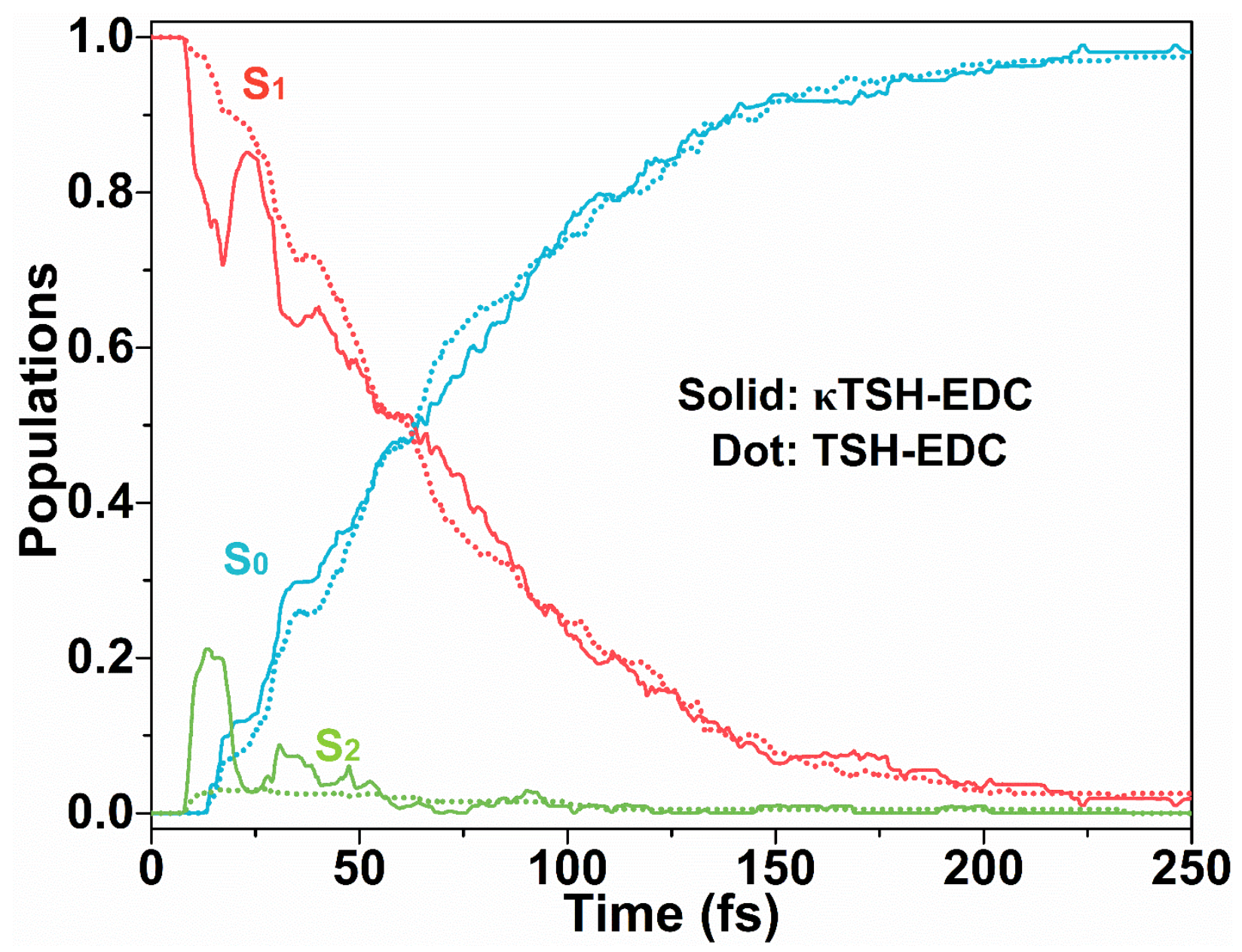

Figure 4. $\mathrm{S}_{0}$ (red), $\mathrm{S}_{1}$ (blue), and $\mathrm{S}_{2}$ (green) populations as functions of time for $\kappa \mathrm{TSH}-\mathrm{EDC}$ (solid) and TSH-EDC (dotted). The curves represent averages over the ensemble of trajectories for the given method. 


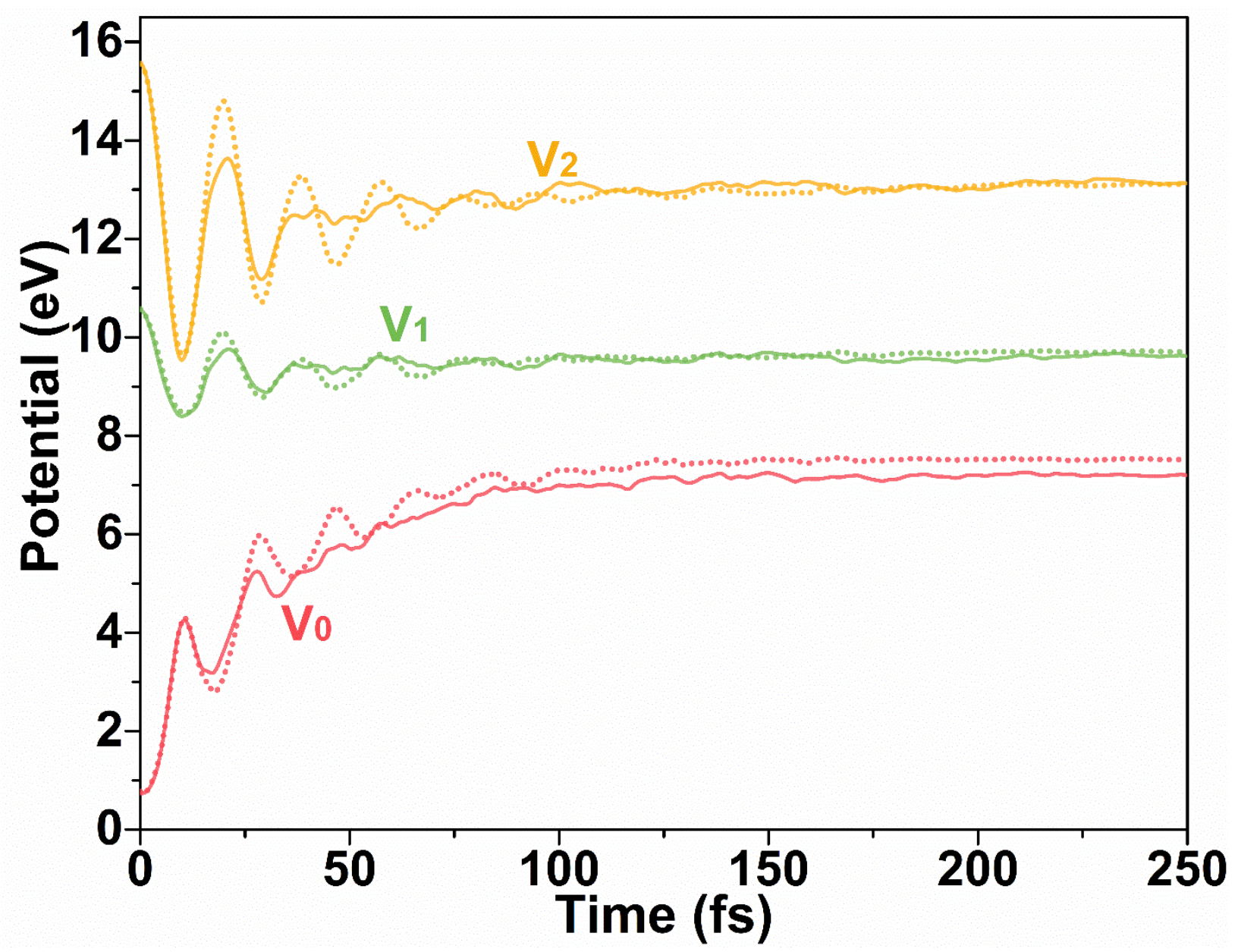

Figure 5. $\mathrm{S}_{0}, \mathrm{~S}_{1}, \mathrm{~S}_{2}$ and mean-field PESs denoted as $V_{0}$ (red), $V_{1}$ (green), $V_{2}$ (yellow), and $V_{\text {eff }}$ (blue) as functions of time for $\mathrm{KTSH}$ (solid) and TSH-EDC (dotted). The curves represent averages over the ensemble of trajectories for the given method. 


\section{口 REFERENCES}

${ }^{1}$ Tully, J. C. Nonadiabatic Processes in Molecular Collisions. In Dynamics of Molecular Collisions, Part B; Miller, W. H., Ed.; Plenum/Springer: New York, 1976; pp. 217-276.

${ }^{2}$ Tully, J. C. Nonadiabatic Dynamics. In Modern Methods for Multidimensional Dynamics Computations in Chemistry, Thompson, D. L.; World Scientific: Singapore, 1998; 34-72.

${ }^{3}$ Worth, G. A.; Cederbaum, L. S. Beyond Born-Oppenheimer: Molecular Dynamics through a Conical Intersection. Annu. Rev. Phys. Chem. 2004, 55, 127-158.

${ }^{4}$ Jasper, A. W.; Zhu, C.; Nangia, S.; Truhlar, D. G. Introductory Lecture: Nonadiabatic Effects in Chemical Dynamics. Faraday Discuss. 2004, 127, 1-22.

${ }^{5}$ Jasper, A.W.; Kendrick, B. K.; Mead, C. A.; Truhlar, D. G. Non-Born-Oppenheimer Chemistry: Potential Surfaces, Couplings, and Dynamics. In Modern Trends in Chemical Reaction Dynamics: Experiment and Theory (Part 1), Yang, X.; Liu, K., Eds.; World Scientific: Singapore, 2004; pp. 329-391.

${ }^{6}$ Computational Photochemistry; Olivucci, M.; Ed.; Elsevier: Amsterdam, 2005.

${ }^{7}$ Chu, T.-S.; Zhang, Y.; Han, K.-L. The Time-Dependent Quantum Wave Packet Approach to the Electronically Nonadiabatic Processes in Chemical Reactions. Int. Rev. Phys. Chem. 2006, 25, 201235.

${ }^{8}$ Conical Intersections: Theory, Computation, and Experiment; Domcke, W., Yarkony, D. R., Köppel, H., Eds.; World Scientific: Singapore, 2011; pp. 375-412.

${ }^{9}$ Tully, J. C. Perspective: Nonadiabatic Dynamics Theory. J. Chem. Phys. 2012, 137, 22 A301.

${ }^{10}$ Plasser, F.; Barbatti, M.; Aquino, A. J. A.; Lischka, H. Electronically Excited States and Photodynamics: A Continuing Challenge. Theor. Chem. Acc. 2012, 131, 1-14.

${ }^{11}$ Curchod, B. F. E.; Rothlisberger, U.; Tavernelli, I. Trajectory-Based Nonadiabatic Dynamics with Time-Dependent Density Functional Theory. ChemPhysChem 2013, 14, 1314-1340.

${ }^{12}$ Persico, M.; Granucci, G. An Overview of Nonadiabatic Dynamics Simulations Methods, with Focus on the Direct Approach versus the Fitting of Potential Energy Surfaces. Theor. Chem. Acc. 2014, 133, 1526.

${ }^{13}$ Zhang, D. H.; Guo, H. Recent Advances in Quantum Dynamics of Bimolecular Reactions, Annu. Rev. Phys. Chem. 2016, 67, 135-158.

${ }^{14}$ Curchod, B. F. E.; Martínez, T. J. Ab Initio Nonadiabatic Quantum Molecular Dynamics. Chem. Rev. 2018, 118, 3305-3336.

${ }^{15}$ Crespo-Otero, R.; Barbatti, M. Recent Advances and Perspectives on Nonadiabatic Mixed Quantum-Classical Dynamics. Chem. Rev. 2018, 118, 7026-7068.

${ }^{16}$ Levine, B. G.; Esch, M. P.; Fales, B. S.; Hardwick, D. T. ; Peng, W.-T.; Shu, Y. Conical Intersections at the Nanoscale: Molecular Ideas for Materials. Annu. Rev. Phys. Chem. 2019, 70, 21-43.

${ }^{17}$ Matsika, S. Electronic Structure Methods for the Description of Nonadiabatic Effects and Conical Intersections. Chem. Rev. 2021, 121, 9407-9449.

${ }^{18}$ Freixas, V. M.; White, A. J.; Nelson, T.; Song, H.; Makhov, D. V.; Shalashilin, D.; FernandezAlberti, S.; Tretiak, S. Nonadiabatic Excited-State Molecular Dynamics Methodologies: Comparison and Convergence. J. Phys. Chem. Lett. 2021, 12, 2970-2982.

${ }^{19}$ Mai, S.; Marquetand, P.; González, L. Nonadiabatic dynamics: The SHARC Approach. WIREs Comput. Mol. Sci. 2018, 8, e1370.

${ }^{20} \mathrm{Xu}$, X.; Zheng, J.; Yang, K. Truhlar, D. G. Photodissociation Dynamics of Phenol: Multistate Trajectory Simulations Including Tunneling. J. Am. Chem. Soc. 2014, 136, 16378-16386. 
${ }^{21}$ Guo, H.; Yarkony, D. R. Accurate Nonadiabatic Dynamics. Phys. Chem. Chem. Phys. 2016, 18, 26335.

${ }^{22}$ Li, S. L.; Truhlar, D. G. Full-Dimensional Multi-State Simulation of the Photodissociation of Thioanisole. J. Chem. Phys. 2017, 147, 044311.

${ }^{23}$ Park, J. W.; Shiozaki, T. On-the-Fly CASPT2 Surface-Hopping Dynamics. J. Chem. Theory Comput. 2017, 13, 3676-3683.

${ }^{24}$ Shu, Y.; Truhlar, D. G. Improved Potential Energy Surfaces of Thioanisole and the Effect of Upper Surface Variations on the Product Distribution Upon Photodissociation. Chem. Phys. 2018, 515, 737-743.

${ }^{25}$ Peng. W.-T.; Fales, B. S.; Shu, Y.; Levine, B. G. Dynamics of Recombination via Conical Intersection in a Semiconductor Nanocrystal. Chem. Sci. 2018, 6, 681-687.

${ }^{26}$ Sifain, A. E.; Bjorgaard, J. A.; Nelson, T. R.; Nebgen, B. T.; White, A. J.; Gifford, B. J.; Gao, D. W.; Prezhdo, O. V.; Fernandez-Alberti, S.; Roitberg, A. E.; Tretiak, S. J. Chem. Theory Comput. 2018, 14, 3955-3966.

${ }^{27}$ Esch. M. P.; Shu, Y.; Levine, B. G. A Conical Intersection Perspective on the Low Nonradiative Recombination Rate in Lead Halide Perovskites. J. Phys. Chem. A 2019, 123, 2661-2673.

${ }^{28}$ Heindl, M.; González, L. A XMS-CASPT2 Non-Adiabatic Dynamics Study on Pyrrole. Comput. Theor. Chem. 2019, 1155, 38-46.

${ }^{29}$ Yu, J. K.; Bannwarth, C.; Liang, R.; Hohenstein, E. G.; Martínez, T. J. Nonadiabatic Dynamics Simulation of the Wavelength-Dependent Photochemistry of Azobenzene Excited to the $n \pi^{*}$ and $\pi \pi^{*}$ Excited States. J. Am. Chem. Soc. 2020, 142, 20680-20690.

${ }^{30}$ De Sio, A.; Sommer, E.; Nguyen, X. T.; Groß, L.; Popović, D.; Nebgen, B. T.; Fernandez-Alberti, S.; Pittalis, S.; Rozzi, C. A.; Molinari, E.; Mena-Osteritz, E.; Bäuerle, P.; Frauenheim, T.; Tretiak, S.; Lienau, C. Intermolecular Conical Intersections in Molecular Aggregates. Nat. Nanotechnol. 2021, 16, 63-68.

${ }^{31}$ Avagliano, D.; Bonfanti, M.; Garavelli, M.; González, L. QM/MM Nonadiabatic Dynamics: the SHARC/COBRAMM Approach. J. Chem. Theory Comput. 2021, 17, 4639-4647.

${ }^{32}$ Child, M. S. Electronic Excitation: Nonadiabatic transitions. In Atom-Molecule Collision Theory: A Guide for the Experimentalist; Bernstein, R. B., Ed.; Plenum: New York, 1979; pp. 427-465.

${ }^{33}$ Tully, J. C.; Preston, R. K. Trajectory Surface Hopping Approach to Nonadiabatic Molecular Collisions: The Reaction of $\mathrm{H}^{+}$with $\mathrm{D}_{2}$. J. Chem. Phys. 1967, 55, 562-572.

${ }^{34}$ Kuntz, P. J.; Kendrick, J.; Whitton, W. N. Surface-Hopping Trajectory Calculations of CollisionInduced Dissociation Processes with and Without Charge Transfer. Chem. Phys. 1979, 38, $147-160$.

${ }^{35}$ Truhlar, D. G.; Duff, J. W.; Blais, N. C.; Tully, J. C.; Garrett, B. C. The Quenching of Na(3 ${ }^{2}$ P) by $\mathrm{H}_{2}$ : Interactions and Dynamics. J. Chem. Phys. 1982, 77, 764-776.

${ }^{36}$ Chapman, S. The classical trajectory surface hopping approach to charge-transfer processes. $A d v$. Chem. Phys. 1992, 82, 423-483.

${ }^{37}$ Tully, J. C. Molecular Dynamics with Electronic Transitions. J. Chem. Phys. 1990, 93, 1061-1071.

${ }^{38}$ Hammes-Schiffer, S.; Tully, J. C. Proton Transfer in Solution: Molecular Dynamics with Quantum Transitions. J. Chem. Phys. 1994, 101, 4657-4667.

${ }^{39}$ Granucci, G.; Persico, M.; Toniolo, A. Direct Semiclassical simulation of photochemical processes with semiempirical wave functions. J. Chem. Phys. 2001, 114, 10608-10615. 
${ }^{40}$ Mandel, A.; Yamijala, S. S.; Huo, P. Quasi-diabatic representation for nonadiabatic propagation. $J$. Chem. Theory Comput. 2018, 14, 1828-1840.

${ }^{41}$ Zhou, W.; Mandal, A.; Huo, P. Quasi-Diabatic Scheme for Nonadiabatic On-the-Fly Simulations. J. Phys. Chem. Lett. 2019, 10, 7062-7070.

${ }^{42}$ Meek, G. A.; Levine, B. G. Accurate and efficient evaluation of transition probabilities at unavoided crossings in ab initio multiple spawning. Chem. Phys. 2015, 460, 117-124.

${ }^{43}$ Meyer, H. D.; Miller, W. H. A Classical Analog for Electronic Degrees of Freedom in Nonadiabatic Collision Processes. J. Chem. Phys. 1979, 70, 3214-3223.

${ }^{44}$ Micha, D. A. A self-consistent Eikonal Treatment of Electronic Transitions in Molecular Collisions. J. Chem. Phys. 1983, 78, 7138-7145.

${ }^{45}$ Kirson, Z.; Gerber, R. B.; Nitzan, A.; Ratner, M. A. Dynamics of Metal Electron Excitation in Atom-Surface Collisions: A Quantum Wave Packet Approach. Surf. Sci. 1984, 137, 527-550.

${ }^{46}$ S.-I. Sawada, A. Nitzan, and H. Metiu, Mean-Trajectory Approximation for Charge- and EnergyTransfer Processes at Surfaces. Phys. Rev. B 1985, 32, 851-867.

${ }^{47}$ Topaler, M. S.; Allison, T. C.; Schwenke, D. W.; Truhlar, D. G. What is the Best Semiclassical Method for Photochemical Dynamics of Systems with Conical Intersections? J. Chem. Phys. 1998, 109, 3321-3345.

${ }^{48}$ Zhu, C.; Nangia, S.; Jasper, A. W.; Truhlar, D. G. Coherent Switching with Decay of Mixing: An Improved Treatment of Electronic Coherence for Non-Born-Oppenheimer Trajectories. J. Chem. Phys. 2004, 121, 7658.

${ }^{49}$ Zhu, C.; Jasper, A. W.; Truhlar, D. G. Non-Born-Oppenheimer Liouville-von Neumann Dynamics. Evolution of a Subsystem Controlled by Linear and Population-Driven Decay of Mixing with Decoherent and Coherent Switching. J. Chem. Phys. 2005, 1, 527-540.

${ }^{50}$ Jasper, A. W.; Nangia, S.; Zhu, C.; Truhlar, D. G. Non-Born-Oppenheimer Molecular Dynamics. Acc. Chem. Res. 2006, 39, 101-108.

${ }^{51}$ Truhlar, D. G. Decoherence in Combined Quantum Mechanical and Classical Mechanical Methods for Dynamics as Illustrated for Non-Born-Oppenheimer Trajectories. In Quantum Dynamics of Complex Molecular Systems, Micha, D. A., Burghardt, I., Eds.; Springer: Berlin, 2007; pp. 227-243.

52 Shu, Y.; Zhang, L.; Mai, S.; Sun, S.; González, L.; Truhlar, D. G.; Implementation of Coherent Switching with Decay of Mixing into SHARC program, J. Chem. Theory Comput., 2020, 16, 34643475.

${ }^{53}$ Shu, Y.; Zhang, L.; Sun, S.; Truhlar, D. G. Time-Derivative Couplings for Self-Consistent Electronically Nonadiabatic Dynamics. J. Chem. Theory Comput. 2020, 16, 4098-4106.

${ }^{54}$ Meek, G. A.; Levine, B. G. Evaluation of the Time-Derivative Coupling for Accurate Electronic Structure State Transition Probabilities from Numerical Simulations. J. Phys. Chem. Lett. 2014, 5, 2351-2356.

55 Shavitt, I. The Method of ConFig.uration Interaction. In Methods of Electronic Structure Theory; Schaefer III, H. F., Ed.; Plenum: New York, 1976; pp. 189-275

56 Čižek, J. On the Use of the Cluster Expansion and the Technique of Diagrams in Calculations of Correlation Effects in Atoms and Molecules. Adv. Chem. Phys. 1969, 14, 35-89.

${ }^{57}$ Koch, H., \& Jørgensen, P. Coupled cluster response functions. J. Chem. Phys. 1990, 93, 33333344. 
${ }^{58}$ Li Manni, G.; Carlson, R. K.; Luo, S.; Ma, D.; Olsen, J.; Truhlar, D. G.; Gagliardi, L. MulticonFig.uration Pair-Density Functional Theory. J. Chem. Theory Comput. 2014, 10, 36693680 .

${ }^{59}$ Bao, J. J.; Zhou, C.; Varga, Z.; Kanchanakungwankul, S.; Gagliardi, L.; Truhlar, D. G. Multi-State Pair-Density Functional Theory. Faraday Discuss. 2020, 224, 348-372.

${ }^{60}$ Bao, J. J.; Zhou, C.; Truhlar, D. G. Compressed-State Multistate Pair-Density Functional Theory. J. Chem. Theory Comput. 2020, 16, 7444-7452.

${ }^{61}$ Lengsfield III, B. H.; Saxe, P.; Yarkony, D. R. On the Evaluation of Nonadiabatic Coupling Matrix Elements Using SA-MCSCF/CI Wave Functions and Analytic Gradient Methods. I. $J$. Chem. Phys. 1984, 81, 4549-4553.

${ }^{62}$ Koch, H.; Jørgensen, P. (1990). Coupled cluster response functions. J. Chem. Phys. 1990, 93, 3333-3344.

${ }^{63}$ Lengsfield III, B. H.; Yarkony, D. R. Nonadiabatic Interactions Between Potential Energy Surfaces: Theory and Applications. Adv. Chem. Phys. 1992, 82, 1-71.

${ }^{64}$ Lomakina, E. I.; Balabin, R. M. Neural Network Approach to Quantum-Chemistry Data: Accurate Prediction of Density Functional Theory Energies. J. Chem. Phys. 2009, 131, 074104.

${ }^{65}$ Behler, J. First Principles Neural Network Potentials for Reactive Simulations of Large Molecular and Condensed Systems. Angew. Chem. Int. Ed. 2017, 56, 12828-12840.

${ }^{66}$ Yao, K.; Herr, J. E.; Toth, D. W.; Mckintyre, R.; Parkhill, J. The TensorMol-0.1 Model Chemistry: A Neural Network Augmented with Long-Range Physics. Chem. Sci. 2018, 9, 2261-2269.

${ }^{67}$ Chandrasekaran, A.; Kamal, D.; Batra, R.; Kim, C.; Chen, L.; Ramprasad, R. Solving the Electronic Structure Problem with Machine Learning. NPJ Comput. Mater. 2019, 5, 22.

${ }^{68}$ Baeck, K. K.; An, H. Practical Approximation of the Non-Adiabatic Coupling Terms for SameSymmetry Interstate Crossings by Using Adiabatic Potential Energies Only. J. Chem. Phys. 2017, 146, 064107.

${ }^{69}$ Mai, S.; Marquetand, P.; González, L.; A General Method to Describe Intersystem Crossing Dynamics in Trajectory Surface Hopping. Int. J. Quantum Chem. 2015, 115, 1215-1231.

${ }^{70}$ Richter, M. Marquetand, P.; González-Vázquez, J.; Sola, I.; González, L. Sharc: Ab Initio Molecular Dynamics with Surface Hopping in the Adiabatic Representation Including Arbitrary Couplings. J. Chem. Theory Comput. 2011, 7, 1253-1258.

${ }^{71}$ Zhang, L.; Shu, Y.; Sun, S.; Truhlar, D. G. Direct Coherent Switching with Decay of Mixing for Intersystem Crossing Dynamics of Thioformaldehyde: The Effect of Decoherence. J. Chem. Phys. 2021, 154, 094310.

${ }^{72}$ Shu, Y.; Zhang, L.; Truhlar, D. G. SHARC-MN-v1.1; University of Minnesota: Minneapolis, 2021), https://comp.chem.umn.edu/sharc-mn (accessed Oct. 27, 2019)

${ }^{73}$ Verlet, L. Computer "experiments" on classical fluids. I. Thermodynamical properties of LennardJones molecules. Phys. Rev. 1967, 159, 98-103.

${ }^{74}$ Topaler, M. S.; Allison, T. C.; Schwenke, D. W.; Truhlar, D. G. Test of Trajectory Surface Hopping Against Accurate Quantum Dynamics for an Electronically Nonadiabatic Chemical Reaction. J. Phys. Chem. A 1998, 102, 1666-1673.

${ }^{75}$ Shu, Y.; L. Zhang; Varga, Z.; Kelsey, P. A.; Kanchanakungwankul, S.; Sun, S.; Truhlar, D. G. Conservation of Angular Momentum in Direct Nonadiabatic Dynamics. J. Phys. Chem. Lett. 2020, $11,1135-1140$. 
${ }^{76}$ Werner, H.-J.; Knowles, P. J.; Knizia, G.; Manby, F. R.; Schütz, M. Molpro: A General-Purpose Quantum Chemistry Program Package. WIREs Comput. Mol. Sci. 2012, 2, 242-253.

${ }^{77}$ Hariharan, P. C.; Pople, J. A. Influence of polarization functions on molecular-orbital hydrogenation energies," Theor. Chem. Acc. 1973, 28, 213-222.

${ }^{78}$ Frisch, M. J.; Head-Gordon, M.; Pople, J. A. A Direct MP2 Gradient Method. Chem. Phys. Lett. 1990, 166, 275-280.

${ }^{79}$ Roos, B. O.; Taylor, P. R. A Complete Active Space SCF Method (CASSCF) Using a DensityMatrix Formulated Super-CI Approach. Chem. Phys. 1980, 48, 157-173.

${ }^{80}$ Werner, H.-J.; Meyer, W. A quadratically convergent MCSCF method for the simultaneous optimization of several states. J. Chem. Phys. 1981, 74, 5794-5801.

${ }^{81}$ Granucci. G.; Persico, M. Critical Appraisal of the Fewest Switches Algorithm for Surface Hopping. J. Chem. Phys. 2007, 126, 134114.

${ }^{82}$ Mai, S.; Richter, M.; Heindl, M.; Menger, M. F. S. J.; Atkins, A.; Ruckenbauer, M.; Plasser, F.; Ibele, L. M.; Kropf, S.; Oppel, M.; Marquetand, P.; González, L. SHARC-v2.1; University of Vienna: Wien, 2019. https://sharc-md.org (accessed Oct. 27, 2019)

Table-of-Contents graphic:

\section{кCSDM, кTSH}

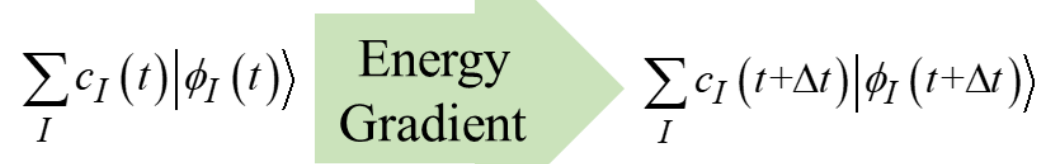

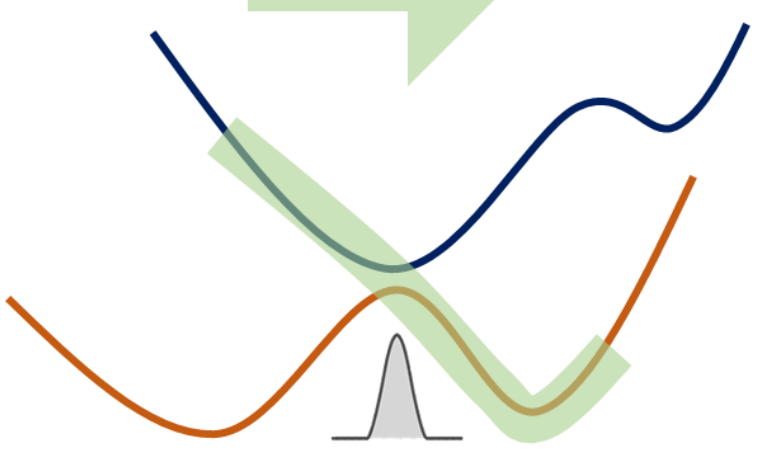


SUPPORTING INFORMATION

OCT. 27, 2021

\section{Nonadiabatic Dynamics Algorithms with Only Potential Energies} and Gradients: Curvature-Driven Coherent Switching with Decay of

\section{Mixing and Curvature-Driven Trajectory Surface Hopping}

Yinan Shu, ${ }^{1, \S}$ Linyao Zhang, ${ }^{2,3, \S}$ Xiye Chen, ${ }^{2,3,}{ }^{*}$ Shaozeng Sun, ${ }^{2}$ Yudong Huang, ${ }^{3}$ and Donald G. Truhlar, ${ }^{1, *}$

${ }^{1}$ Department of Chemistry and Supercomputing Institute, University of Minnesota, Minneapolis, MN 55455-0431, USA

${ }^{2}$ School of Energy Science and Engineering, Harbin Institute of Technology,

Harbin 150001, P. R. China

${ }^{3}$ School of Chemistry and Chemical Engineering, Harbin Institute of Technology, Harbin 150001, P. R. China

*Corresponding authors: truhlar@umn.edu, chenxiye@hit.edu.cn 


\section{Contents}

Optimized geometry of ethylene with MP2/6-31G** ........................... S-3

SHARC-MN input file for $\boldsymbol{\kappa} C$ SDM calculation..................................... S-3

SHARC-MN input file for tCSDM calculation................................... S-4

SHARC-MN input file for CSDM calculation ..................................... S-5

SHARC-MN input file for $\mathbf{~}$ TSH-EDC calculation .............................. S-6

SHARC-MN input file for TSH-EDC(TDC) calculation ....................... S-7

SHARC-MN input file for TSH-EDC(NAC) calculation ..................... S-8

Pointer state population for CSDM methods..................................... S-7

Pointer state population for TSH methods ......................................... S-8 
Optimized geometry of ethylene with $\mathrm{MP2} / 6-31 \mathrm{G} * *$

$\begin{array}{lrcc}\mathrm{C} & 0.1253759125 & -0.2973946268 & 0.0548520616 \\ \mathrm{C} & 0.0457468876 & 0.0009727000 & 1.3551368234 \\ \mathrm{H} & 1.0405529177 & -0.6681691515 & -0.3858045654 \\ \mathrm{H} & -0.7223227760 & -0.1792792761 & -0.6059533265 \\ \mathrm{H} & -0.8694303378 & 0.3717448374 & 1.7958029647 \\ \mathrm{H} & 0.8934373960 & -0.1170394830 & 2.0159700422\end{array}$

SHARC-MN input file for $\kappa C S D M$ calculation

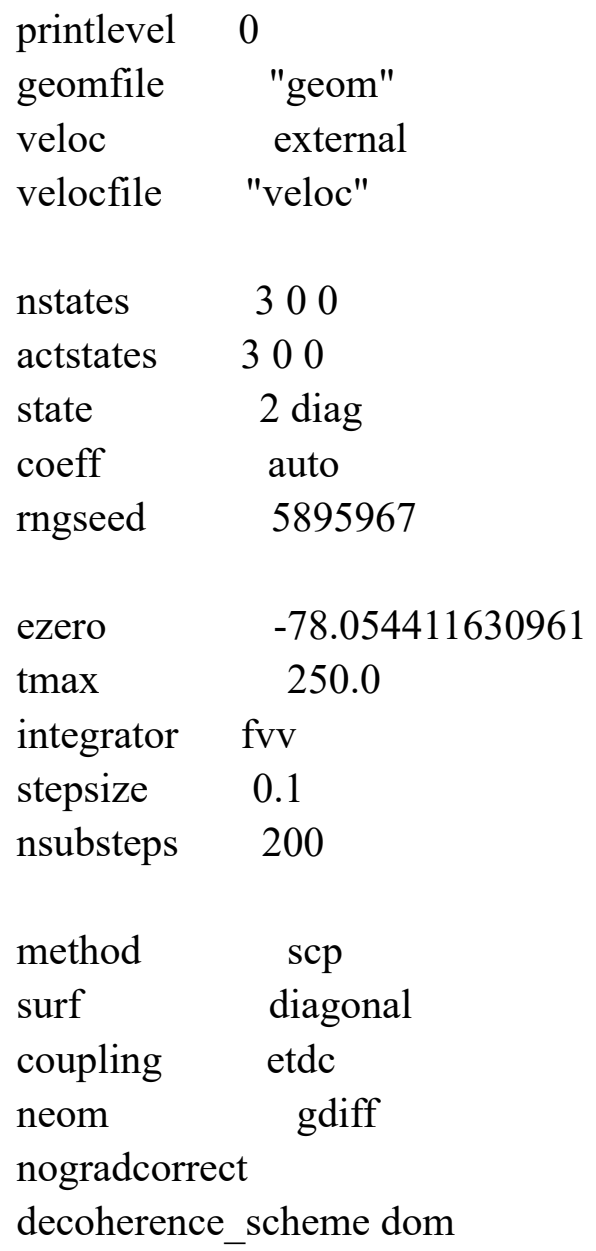


SHARC-MN input file for tCSDM calculation

$\begin{array}{lc}\begin{array}{l}\text { printlevel } \\ \text { geomfile }\end{array} & \begin{array}{c}\text { "geom" } \\ \text { external }\end{array} \\ \begin{array}{l}\text { veloc } \\ \text { velocfile }\end{array} & \text { "veloc" } \\ \text { nstates } & 300 \\ \text { actstates } & 300 \\ \text { state } & 2 \text { diag } \\ \text { coeff } & \text { auto } \\ \text { rngseed } & 5895967 \\ & \\ \text { ezero } & -78.054411630961 \\ \text { tmax } & 250.0 \\ \text { integrator } & \text { fvv } \\ \text { stepsize } & 0.1 \\ \text { nsubsteps } & 200 \\ & \\ \text { method } & \text { scp } \\ \text { surf } & \text { sharc } \\ \text { coupling } & \text { overlap } \\ \text { nogradcorrect } & \\ \text { decoherence_scheme dom } & \end{array}$


SHARC-MN input file for CSDM calculation

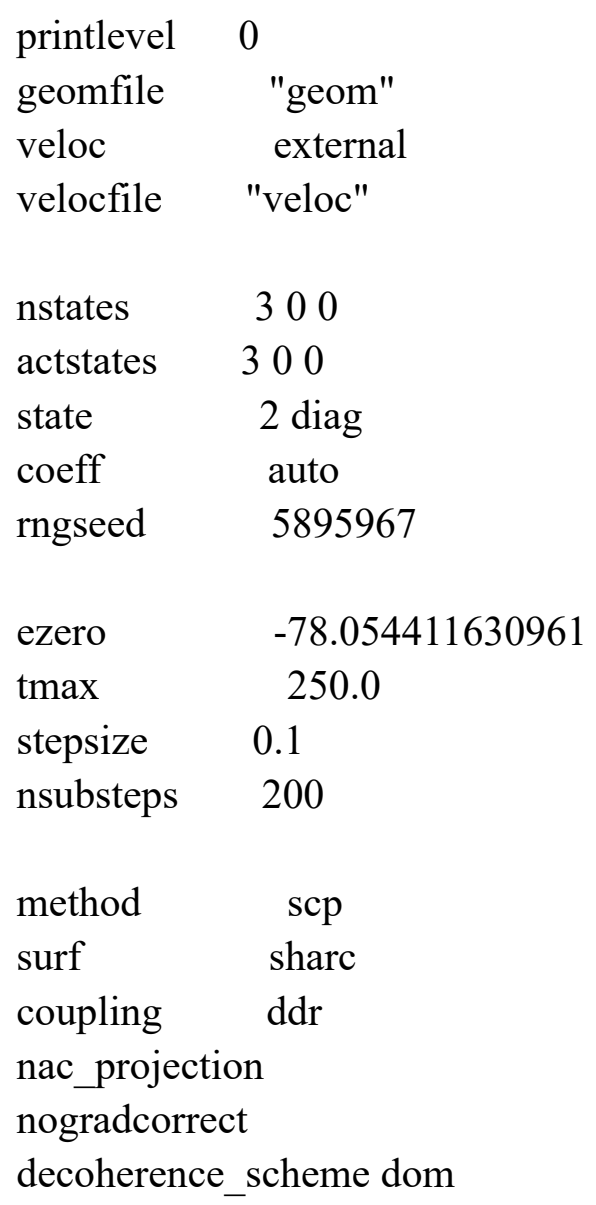


SHARC-MN input file for $\kappa$ TSH-EDC calculation

\begin{tabular}{|c|c|}
\hline $\begin{array}{l}\text { printlevel } \\
\text { geomfile } \\
\text { veloc } \\
\text { velocfile }\end{array}$ & $\begin{array}{l}0 \\
\text { "geom" } \\
\text { external } \\
\text { "veloc" }\end{array}$ \\
\hline nstates & 300 \\
\hline actstates & 300 \\
\hline state & 2 diag \\
\hline coeff & auto \\
\hline rngseed & 5895967 \\
\hline ezero & -78.054411630961 \\
\hline $\operatorname{tmax}$ & 250.0 \\
\hline integrator & fvv \\
\hline stepsize & 0.1 \\
\hline nsubsteps & 200 \\
\hline $\begin{array}{l}\text { method } \\
\text { surf } \\
\text { coupling } \\
\text { ekincorrect } \\
\text { decoherence } \\
\text { nogradcorre } \\
\text { grad_select }\end{array}$ & $\begin{array}{l}\text { tsh } \\
\text { diagonal } \\
\text { etdc } \\
\text { parallel_diff } \\
\text { scheme edc } \\
\text { ct }\end{array}$ \\
\hline
\end{tabular}


SHARC-MN input file for TSH-EDC(TDC) calculation

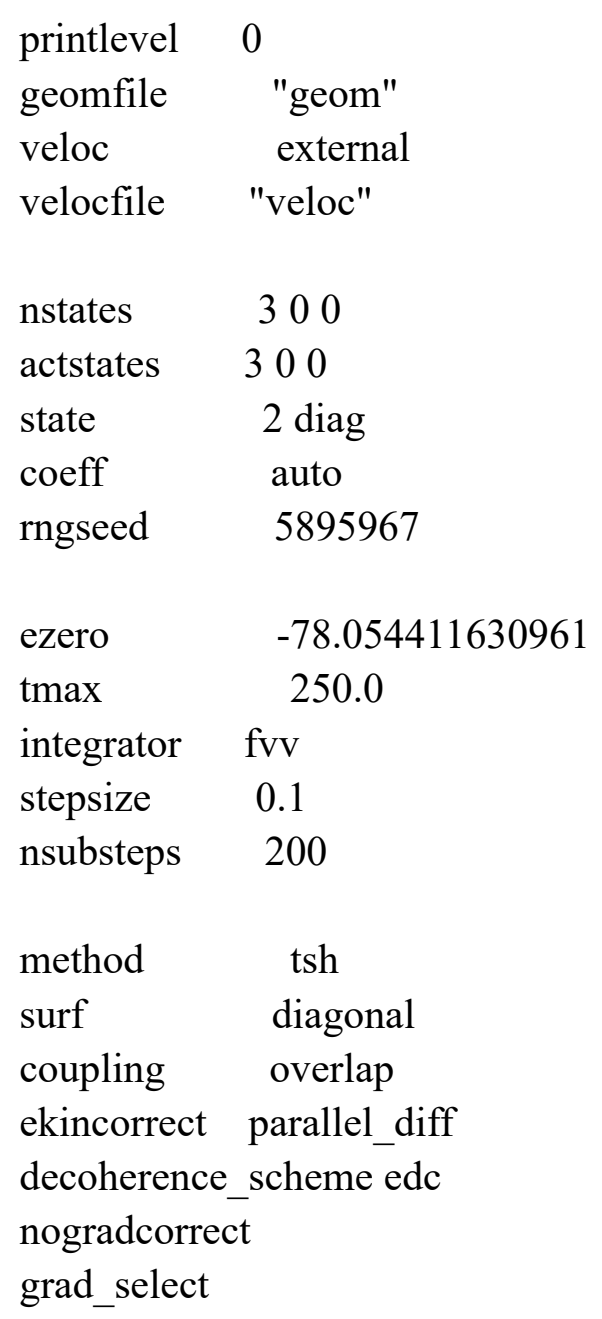


SHARC-MN input file for TSH-EDC(NAC) calculation

\begin{tabular}{|c|c|}
\hline $\begin{array}{l}\text { printlevel } \\
\text { geomfile } \\
\text { veloc } \\
\text { velocfile }\end{array}$ & $\begin{array}{l}0 \\
\text { "geom" } \\
\text { external } \\
\text { "veloc" }\end{array}$ \\
\hline nstates & 300 \\
\hline actstates & 300 \\
\hline state & 2 diag \\
\hline coeff & auto \\
\hline rngseed & 5895967 \\
\hline ezero & -78.054411630961 \\
\hline $\operatorname{tmax}$ & 250.0 \\
\hline stepsize & 0.1 \\
\hline nsubsteps & 200 \\
\hline $\begin{array}{l}\text { method } \\
\text { surf } \\
\text { coupling } \\
\text { ekincorrect } \\
\text { nac_project } \\
\text { nogradcorre } \\
\text { decoherenc } \\
\text { grad_select } \\
\text { nac_select }\end{array}$ & $\begin{array}{l}\text { tsh } \\
\text { sharc } \\
\text { ddr } \\
\text { parallel_pnac } \\
\text { on } \\
\text { ct } \\
\text { scheme edc }\end{array}$ \\
\hline
\end{tabular}




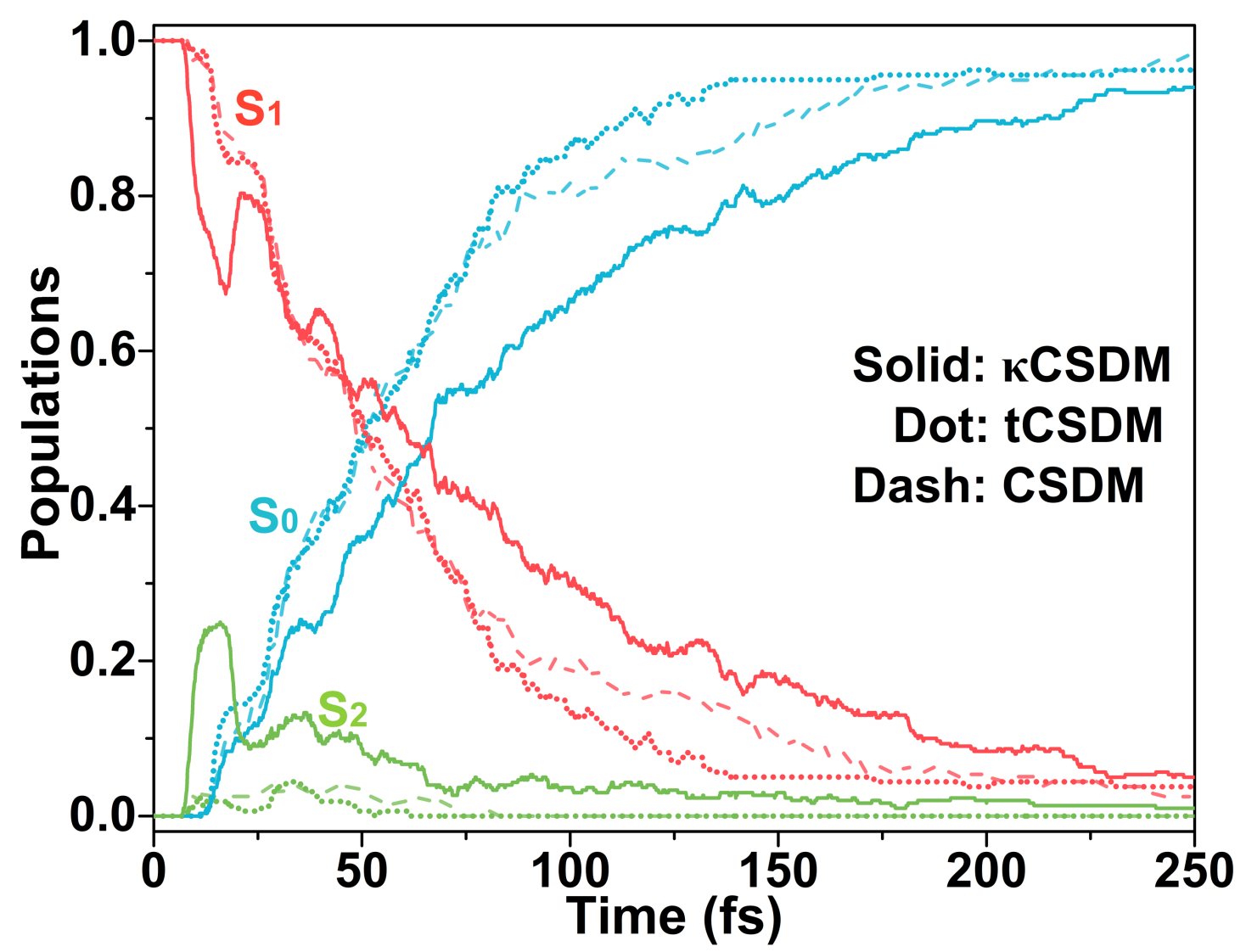

Figure $\mathrm{S} 1 . \mathrm{S}_{0}, \mathrm{~S}_{1}$, and $\mathrm{S}_{2}$ populations as a function of time are shown in red, blue, and green colors respectively for $\kappa \mathrm{CSDM}$ (solid), tCSDM (dot) and CSDM (dash) trajectory ensemble results. At 250 fs the $\mathrm{S}_{0}, \mathrm{~S}_{1}$, and $\mathrm{S}_{2}$ pointer state populations are 0.94, 0.05, 0.01 for $\kappa \mathrm{CSDM}, 0.96,0.04,0.00$ for tCSDM, and $0.97,0.03,0.00$ for CSDM respectively. The half-lives time $\tau_{1 / 2}$ obtained from the simulations for $\mathrm{S}_{1}$ excited state are 59,51 , and 51 fs for $\kappa$ CSDM, tCSDM, CSDM, respectively. 


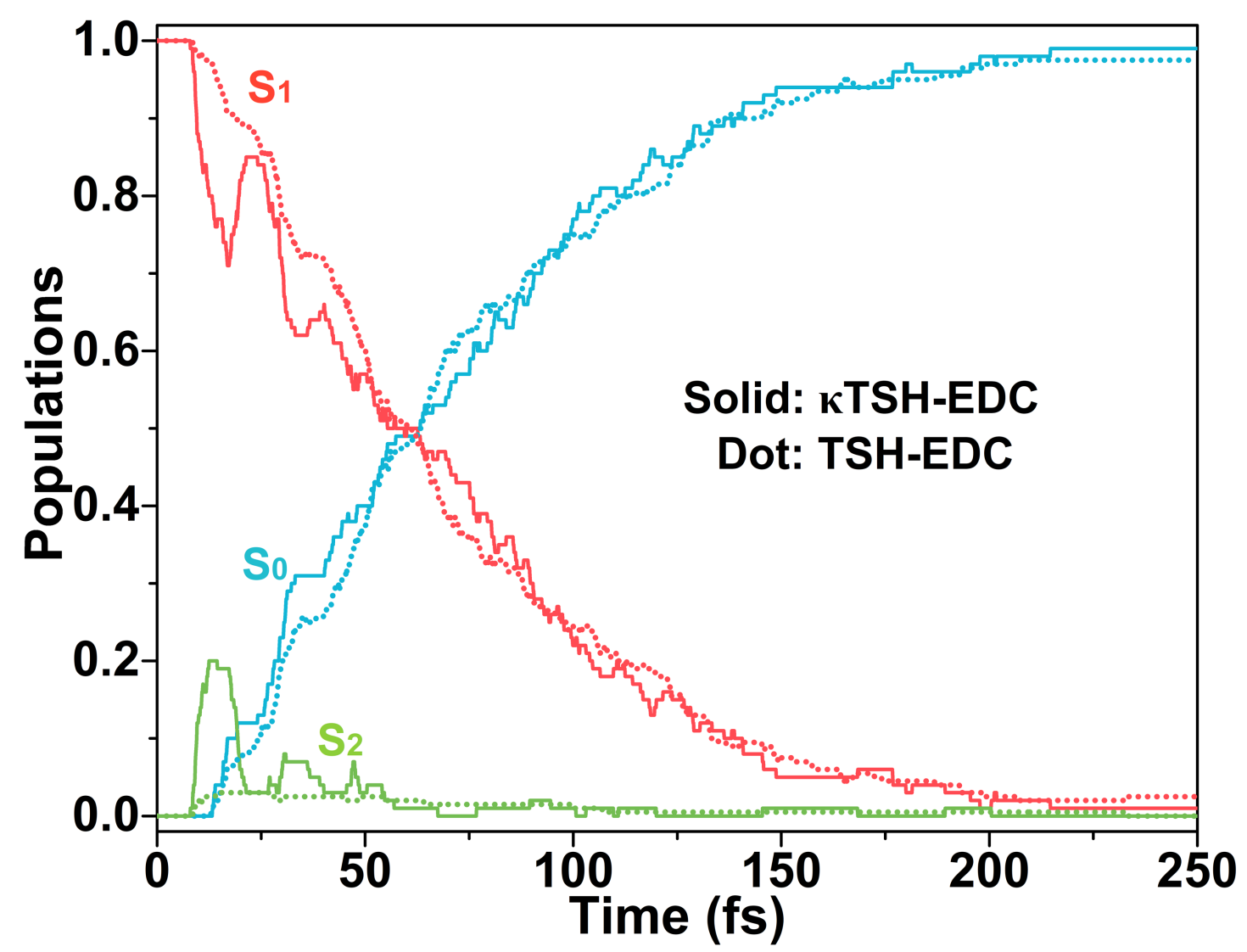

Figure $\mathrm{S}_{2} . \mathrm{S}_{0}, \mathrm{~S}_{1}$, and $\mathrm{S}_{2}$ populations as a function of time are shown in red, blue, and green colors respectively for $\kappa$ TSH (solid) and TSH-EDC (dotted). The half-lives time $\tau_{1 / 2}$ obtained from the simulations for $S_{1}$ excited state are 63 and 61 fs for $\kappa$ TSH and TSH, respectively. 